\title{
Development of an exosomal biomarker signature to detect minimal residual disease in dogs with osteosarcoma using a novel xenograft platform and machine learning
}

Kelly M. Makielski ${ }^{1,2,3, *}$, Alicia J. Donnelly ${ }^{1,2,3, *, a}$, Ali Khammanivong ${ }^{1,2,3, *}$, Milcah C. Scott $^{1,2,3, b}$, Andrea R. Ortiz ${ }^{4, c}$, Dana C. Galvan ${ }^{4, d}$, Hirotaka Tomiyasu ${ }^{1,2,3, e}$, Clarissa Amaya $^{4, f}$, Kristi Ward ${ }^{4, g}$, Alexa Montoya ${ }^{4, h}$, John R. Garbe ${ }^{5,6}$, Lauren J. Mills ${ }^{3,5,7}$, Gary R. Cutter $^{8}$, Joelle M. Fenger ${ }^{9,10, i}$, William C. Kisseberth ${ }^{9,10}$, Timothy D. O'Brien ${ }^{1,11,14}$, Logan G. Spector $1,3,7,12$, Brad A. Bryan ${ }^{4, j}$, Subbaya Subramanian ${ }^{1,3,13}$, Jaime F. Modiano $1,2,3,14,15,16, \wedge$

* Co-first authors

${ }^{\wedge}$ Corresponding author

\section{Institutional Affiliations:}

${ }^{1}$ Animal Cancer Care and Research Program, University of Minnesota, St. Paul, MN, USA

${ }^{2}$ Department of Veterinary Clinical Sciences, College of Veterinary Medicine, University of Minnesota, St. Paul, MN, USA

${ }^{3}$ Masonic Cancer Center, University of Minnesota, Minneapolis, MN, USA

${ }^{4}$ Texas Tech Health Sciences Center, EI Paso, TX, USA

${ }^{5}$ Minnesota Supercomputing Institute, University of Minnesota, Minneapolis, MN, USA

${ }^{6}$ University of Minnesota Genomics Center, University of Minnesota, Minneapolis, MN, USA

${ }^{7}$ Department of Pediatrics, University of Minnesota Medical School, Minneapolis, MN, USA

${ }^{8}$ Department of Biostatistics, University of Alabama, Birmingham, Birmingham, AL, USA ${ }^{9}$ Department of Veterinary Clinical Sciences, College of Veterinary Medicine, The Ohio State University, Columbus, OH, USA

${ }^{10}$ Arthur G. James The Ohio State University Comprehensive Cancer Center, The Ohio State University, Columbus, OH, USA

${ }^{11}$ Department of Veterinary Population Medicine, University of Minnesota, Minneapolis, MN, USA

12 Division of Epidemiology and Clinical Research, University of Minnesota, Minneapolis, MN, USA

${ }^{13}$ Department of Surgery, University of Minnesota Medical School, Minneapolis, MN, USA

${ }^{14}$ Stem Cell Institute, University of Minnesota, Minneapolis, MN, USA

${ }^{15}$ Center for Immunology, University of Minnesota, Minneapolis, MN, USA

${ }^{16}$ Department of Laboratory Medicine and Pathology, University of Minnesota Medical School, Minneapolis, MN, USA 


\section{Current addresses for authors:}

a Dr. Alicia Donnelly - Senior Research Veterinarian, The Children's Hospital of Philadelphia Research Institute, 3501 Civic Center Blvd, Philadelphia, PA 19104; donnellya3@chop.edu

${ }^{b}$ Milcah Scott - University of Minnesota, Microbiology, Immunology, and Cancer Biology Graduate Program; Minneapolis, MN, USA; scot0340@umn.edu

${ }^{c}$ Andrea Ortiz - Baylor College of Medicine, Cellular and Molecular and Cellular Biology; Houston, TX, andreao@bcm.edu

d Dana Galvan -University of New Mexico, Department of Radiology, 2211 Lomas Blvd NE, Albuquerque, NM 87106; dcgalvan@salud.unm.edu

e Dr. Hirotaka Tomiyasu - Department of Veterinary Internal Medicine, Graduate School of Agricultural and Life Sciences, The University of Tokyo, Tokyo, Japan;

hirotaka.tomiyasu@gmail.com

f Clarissa Amaya - HCA Healthcare, Graduate Medical Education, clarissa.amaya3@gmail.com

9 Kristi Ward - University of Virginia, Radiation Oncology, https://med.virginia.edu/radiation-oncology/education-programs/medical-residencyprogram/residents/kristin-ward-md/; kaw9na@hscmail.mcc.virginia.edu

${ }^{h}$ Alexa Montoya - University of Texas, El Paso, Biology, anmontoya@miners.utep.edu i Dr. Joelle Fenger - 601 Vernon L Tharp St, Columbus, OH, USA; fengerjm@gmail.com

jDr. Brad Bryan - private consulting; Bradallenbryan@gmail.com

\section{Funding:}

This work was supported in part by grants D15CA-047 (to JFM, BAB, SS, TDO) and D13CA-032 (to JFM and SS) from Morris Animal Foundation, CA170218 (to JFM and SS) from the Congressionally-designated Medical Research Program of the Department of Defense, MNP \#15.25 (to JFM) from the Minnesota Partnership for Biotechnology and Medical Genomics, RSG-13-381-01 (to SS) from the American Cancer Society, 2011-1 (to JFM and SS) from the Karen Wykoff Rein in Sarcoma Foundation.

Philanthropic support for this project included directed grants from the Nat Fund of the Children's Cancer Research Fund (to JFM and LGS), GREYlong (to JFM), the Skippy Frank Fund for Life Sciences and Translational Research (JFM), as well as unrestricted gifts from public and anonymous donors supporting the Animal Cancer Care and Research Program of the University of Minnesota. KMM was supported in part by a postdoctoral fellowship from the institutional training grant in Molecular, Genetic, and Cellular Targets of Cancer (T32 CA009138). AJD was supported in part by a postdoctoral fellowship from Morris Animal Foundation (D16CA-405). JFM was supported in part by the Alvin and June Perlman Endowed Chair in Animal Oncology. Portions of this work were conducted in the Minnesota Nano Center, which is supported by the National Science Foundation through the National Nano Coordinated Infrastructure Network (NNCI) under Award Number ECCS-2025124. Sample collection was supported in part by the Ohio State University CTSA grant UL1TR002733 from the National Center for Advancing Translational Sciences and by the Ohio State Cancer Center designated cancer center support grant P30 CA016058. Support from bioinformatics was provided by the Masonic Cancer Center designated cancer center 
bioRxiv preprint doi: https://doi.org/10.1101/2021.02 11.429432; this version posted February 12, 2021. The copyright holder for this preprint

(which was not certified by peer review) is the author/funder, who has granted bioRxiv a license to display the preprint in perpetuity. It is made available under aCC-BY-NC-ND 4.0 International license.

support grant P30 CA077598. The content of this manuscript is solely the responsibility of the authors and does not necessarily represent the official views of any of the funding agencies listed above. 


\section{Abstract}

Osteosarcoma has a guarded prognosis. A major hurdle in developing more effective osteosarcoma therapies is the lack of disease-specific biomarkers to predict risk, prognosis, or therapeutic response. Exosomes are secreted extracellular microvesicles emerging as powerful diagnostic tools. However, their clinical application is precluded by challenges in identifying disease-associated cargo from the vastly larger background of normal exosome cargo. We developed a method using canine osteosarcoma in mouse xenografts to distinguish tumor-derived from host-response exosomal mRNAs. The model allows for the identification of canine osteosarcoma-specific gene signatures by RNA sequencing and a species-differentiating bioinformatics pipeline. An osteosarcoma-associated signature consisting of five gene transcripts (SKA2, NEU1, $P A F 1, P S M G 2$, and NOB1) was validated in dogs with spontaneous osteosarcoma by qRT-PCR, while a machine learning model assigned dogs into healthy or disease groups. Serum/plasma exosomes were isolated from 53 dogs in distinct clinical groups ("healthy", "osteosarcoma", "other bone tumor", or "non-neoplastic disease"). Pretreatment samples from osteosarcoma cases were used as the training set and a validation set from post-treatment samples was used for testing, classifying as "osteosarcoma-detected" or "osteosarcoma-NOT detected". Dogs in a validation set whose post-treatment samples were classified as "osteosarcoma-NOT detected" had longer remissions, up to 15 months after treatment. In conclusion, we identified a gene signature predictive of molecular remissions with potential applications in the early detection and minimal residual disease settings. These results provide proof-of-concept 
for our discovery platform and its utilization in future studies to inform cancer risk, diagnosis, prognosis, and therapeutic response.

\section{Introduction}

Osteosarcoma is a rare disease that disproportionately affects children, adolescents, and young adults (1). More than half of osteosarcoma patients relapse and die from metastatic disease within 10 years of their initial diagnosis $(1,2)$, highlighting the need for predictive biomarkers to personalize therapies. Previously, we have identified evolutionarily conserved transcriptional programs with high prognostic value; however, practical obstacles have prevented their wide adoption into clinical practice (3-5). Thus, it is apparent that non-invasive tests that inform prognosis and longitudinal remission status represent a continued unmet need for osteosarcoma patients.

Serum exosomes can be used to address these unmet needs in osteosarcoma $(6,7)$.

Exosomes are secreted, membrane-bound vesicles measuring 30 to $200 \mathrm{nM}$ in diameter that originate from the fusion of multivesicular endosomes to the plasma membrane (8). Like other microvesicles, exosomal cargo includes RNA, DNA, proteins, lipids, and cellular metabolites. Exosomes can be powerful diagnostic tools. Specifically, they are stable in biological fluids, can be efficiently and non-invasively isolated, and contain cargo that can be significantly associated with disease states (9-11). 
Furthermore, the utility of serum exosomes as a diagnostic/prognostic platform is independent of the source and function of such cargo. There have been recent gains in enrichment of exosomes and/or comparably sized microvesicles from blood, plasma, and serum using instrumentation and methodology that is routinely available in diagnostic laboratories (9-11), allowing us to envision applications of exosome diagnostics as a realistic goal. However, the identification and differentiation of cargo originating from diseased cells (signal) from the background of normal exosomes (noise) is still a major obstacle that precludes wide use of exosomes in clinical laboratory medicine.

Previously, we developed a method to identify species-specific messenger RNA (mRNA) sequences in tumor xenografts (tumor, or donor species, and stroma, or host species) $(12,13)$. In the present study, we further extended that method to serum exosomes, allowing for identification of a tumor-specific five-gene signature that accurately discriminates osteosarcoma tumor-bearing dogs from dogs in other disease categories and dogs free of apparent disease. Individually, none of the genes could reliably predict the presence of osteosarcoma or minimal residual disease, but when combined with machine learning the combined five-gene signature could accurately classify osteosarcoma and non-osteosarcoma in dogs undergoing treatment for this disease. Overall, we demonstrate the discovery of exosome-based biomarkers that have the potential to predict tumor biological behavior, and thus inform prognosis and guide treatment of osteosarcoma in dogs, providing proof of concept to develop and apply comparable approaches for human osteosarcoma patients. 


\section{Materials and Methods}

Cell culture. Two canine osteosarcoma cell lines, representing previously described "highly aggressive" and "less aggressive" molecular phenotypes (OS-1 and OS-2), were used in this study (14). OS-1 and OS-2 are derivatives of the OSCA-40 (Kerafast, Inc., catalog \#EMN003) and OSCA-32 cell lines (Kerafast, Inc., Boston, MA, catalog \#EMN002), respectively. OS-1 and OS-2 cells were modified to stably express green fluorescent protein (GFP; ThermoFisher Scientific, Waltham, MA) and firefly luciferase (ThermoFisher Scientific, Waltham, MA) and used for orthotopic injections in mice. Prior to mouse injections, cells were grown in exosome-depleted DMEM media (DMEM with $5 \%$ glucose and L-glutamine (GIBCO, ThermoFisher Scientific, Waltham, MA, catalog \#11965) supplemented with 10\% exosome-depleted FBS Media Supplement - USA Certified (SBI, Palo Alto, CA, catalog \# EXO-FBS-250A-1), 10mM 4-(2-hydroxyethyl)-1piperazine ethanesulphonic acid buffer (HEPES; ThermoFisher Scientific, Waltham, MA, catalog \#15630) and 0.1\% Primocin (Invivogen, San Diego, CA, catalog \#ant-pm1)), and cultured at $37^{\circ} \mathrm{C}$ in a humidified atmosphere of $5 \% \mathrm{CO}_{2}$. Each cell line was passaged more than 15 times before the experiments; however, cell lines were repeatedly authenticated at regular intervals (IDEXX BioResearch, Columbia, MO) during experimentation based on short tandem repeats.

Primary cultures of human pulmonary microvascular endothelial cells (Lonza, Walkersville, MD, catalog \#CC-2527) were grown in EGM-2 Endothelial Cell Growth Medium-2 Bullet kit (Lonza). Primary cultures of human pulmonary fibroblasts (Lonza, catalog \#CC-2512) were cultured in FGM-2 Fibroblast Cell Growth Medium-2 Bullet kit (Lonza). For exosome depleted conditions, the FBS aliquot in each Bullet kit was excluded from the growth media. Cell lines were authenticated at regular intervals (IDEXX BioResearch, Columbia, MO), based on short tandem repeats.

Exosome purification. Cells were cultured in exosome depleted media and exosomes were isolated using the ExoQuick TC kit (SBI, Palo Alto, CA, catalog \#EXOTC10A-1) according to the manufacturer's instructions. 
Immunohistochemistry and immunofluorescence. Human osteosarcoma tissue microarrays were obtained from US Biomax (Rockville, MD, catalog \#OS804), and antigens were detected using immunohistochemistry and quantified by previously described methodology (15). For immunofluorescence, cells were treated as indicated and fixed in ice-cold paraformaldehyde solution. Immunofluorescent detection of CD9, CD63, and CD81 was performed using the EXOAB-KIT-1 (SBI, Palo Alto, CA, catalog \#EXOAB-KIT-1) and anti-phalloidin-conjugated secondary antibodies. DAPI counter staining was used as a nuclear stain. The anti-human tetraspanin antibodies used for immunohistochemistry and immunofluorescence cross-react against the canine proteins, as determined by immunoblotting where each antibody recognizes unique proteins with the correct electrophoretic mobility (Supplemental Figure S3, and data not shown).

Electron microscopy. Exosomes were isolated from OSCA-40 cells using the ExoQuick TC protocol, fixed in 2.5\% paraformaldehyde, and washed in PBS. The exosomes were suspended in Milli-Q water, immediately applied to a glass slide, and allowed to air dry for 1 hour. The slides were dehydrated with ethanol, sputtered coated with a gold layer, and imaged using a Zeiss EVO scanning electron microscope.

Immunoblotting. 200 $\mu$ l of Pierce RIPA buffer (ThermoFisher Scientific, Waltham, MA, catalog \#89900) combined with $1 \mu$ of HALT protease inhibitor and $1 \mu$ of HALT phosphatase inhibitor cocktail (ThermoFisher Scientific, Waltham, MA, catalog \#78420) were added to cell or exosome pellets and vortexed for $\sim 15$ seconds. The samples were placed at room temperature for 5 minutes to allow complete lysis before pre-clearing nuclei and insoluble material by centrifugation. The protein content was determined using the BCA assay kit as recommended by the manufacturer (ThermoFisher Scientific, Waltham, MA, catalog \#23225). For immunoblotting, 50 $\mu$ g of protein for each sample were diluted into, Laemmli buffer, heated at $95^{\circ} \mathrm{C}$, and then immediately chilled on ice before loading onto the gels. SDS-PAGE electrophoresis and transfer to PVDF membranes was done using routine protocols (16). Membranes were blocked with $5 \%$ 
dry milk in Tris Buffered Saline containing 0.05\% Tween (TBS-T), followed by overnight incubation at $4^{\circ} \mathrm{C}$ with antibodies directed against CD9, CD63, and CD81 (SBI, Palo Alto, CA, catalog \#EXOAB-KIT-1) at 1:1000 dilution in TBS-T buffer containing $5 \%$ dry milk. Anti- $\beta$ actin was used as described (17) to serve as a control for depletion of cytosolic proteins in exosomes. Blots were washed and incubated for one hour at room temperature with a secondary goat anti-rabbit-HRP antibody at 1:20,000 dilution. The blots were finally incubated with chemiluminescence substrate and visualized on a LICOR Odyssey Imager (LI-COR, Lincoln, NE).

Nanoparticle tracking. Exosomes were enriched from samples as detailed above, and resuspended in PBS to a total volume of $1 \mathrm{ml}$. The size distribution of extracellular vesicles was measured using a NanoSight Nanoparticle Tracking Analyzer (Salisbury, United Kingdom), using the settings recommended by the manufacturer. Size, frequency, and distribution measurements were recorded in triplicate for each sample and were analyzed by the built-in NanoSight Software.

Plasmids and transfection. Transduction of osteosarcoma cells was performed using the pCT-CMV-GFP-MCS-EF1a-Puro lentiviral system (SBI, Palo Alto, CA, catalog \#CYTO800-PA-1) as described (18). Puromycin was used to select stably expressing cells, and cells were grown in exosome depleted media prior to exosome collection.

Tumor xenografts. Six week-old, female, athymic nude mice (strain NCr nu/nu) were obtained from Charles River Laboratories (Wilmington, MA). Animals were assigned to separate cages in random order for each experiment. All mouse experiments were approved by The University of Minnesota Institutional Animal Care and Use Committee (Protocols 1307-30806A, 1606-33857A, and 1803-35710A). For intratibial injections, mice were anesthetized with xylazine (10 mg/kg, intraperitoneally (I.P.)) and ketamine (100mg/kg, I.P.) in preparation for intratibial (I.T.) injections. Canine osteosarcoma cells were suspended in sterile PBS (ThermoFisher Scientific, Waltham, MA, catalog \#10010049) and 10 $\mu$ l containing $1 \times 10^{5}$ cells was injected I.T. as previously reported $(12,17)$. Control mice had $10 \mu$ l sterile PBS injected I.T. All injections were administered 
into the left tibia using a tuberculin syringe with 29-gauge needle. For each osteosarcoma cell line, OS-1 and OS-2, five mice received cell-I.T. injections; 3 mice received sham (PBS)-I.T. injections. Buprenorphine $(0.075 \mathrm{mg} / \mathrm{kg}$, I.P. every 8 hours (Reckitt Benckiser Healthcare, Richmond, VA)) was administered for analgesia for 24 hours following the injections, and prophylactic ibuprofen was administrated in the water for the next 3 days. Mice were monitored by weekly bioluminescence imaging and tumor size measurements. At 8 weeks after the injections, the mice were humanely euthanized using a barbiturate overdose. Blood was collected via intracardiac phlebotomy. The tibiae and the lungs were collected from mice injected with osteosarcoma cells $(n=10)$ and placed in $10 \%$ neutral buffered formalin for histopathology or stored at $-80^{\circ} \mathrm{C}$. The presence of tumors was confirmed histologically.

Osteosarcoma xenograft serum exosome precipitation and RNA extraction. Exosomes were precipitated from serum samples from control mice and from tumor bearing mice at week-8 using ExoQuick reagent (SBI, Palo Alto, CA, catalog \#EXOQ5A-1) according to the manufacturer's instructions. Briefly, serum was mixed with ExoQuick reagent at a volume of $252 \mu \mathrm{l}$ ExoQuick per $1 \mathrm{ml}$ of serum. The mixture was incubated for 30 minutes at $4^{\circ} \mathrm{C}$, followed by centrifugation at $1,500 \times \mathrm{g}$ for 30 minutes to precipitate exosomes. The resulting supernatant was removed and discarded, and the tubes were centrifuged for an additional 5 minutes at $1,500 \times \mathrm{g}$ to remove any remaining supernatant. Exosomal RNA was extracted using SeraMir ExoRNA Amp Kit (SBI, Palo Alto, CA, catalog \#RA800A-1), according to the manufacturer's instructions.

Library preparation and next-generation sequencing. Pooled serum from each group was sequenced and analyzed. Sequencing libraries were prepared using the Clontech SMARTer® Stranded Total RNA-Seq Kit v2 - Pico Input Mammalian kit (Takara Bio, Kasatsu, Japan). RNA sequencing (50-bp paired-end, with HiSeq 2500 Illumina) was performed at the University of Minnesota Genomics Center (UMGC). A minimum of 16 million read-pairs was generated for each sample and the average quality scores were above Q30 for all pass-filter reads. 
Bioinformatics analysis. Initial quality control analysis of RNA sequencing FASTQ data was performed using FastQC software (v0.11.5). FASTQ data were trimmed with Trimmomatic (v0.33.0). Kallisto (v0.43.0) was used for pseudoalignment and quantifying transcript abundance. For accurate alignment of sequencing reads to canine and murine genes within xenograft tumors, a kallisto index was built from a multi-sequence FASTA file containing both the canine (CanFam3.1) and murine (GRCm38.p5) genomes. For each species, transcripts <200bp were removed from the FASTA files. The masked FASTA files were then merged for a total of 121,749 murine and canine transcripts. Insertion size metrics were calculated for each sample using Picard software (v1.126). Data will be deposited in GenBank/GEO. The 'DESeq2' package in RStudio was used for differential analysis of transcript counts obtained from kallisto data. Transcript counts were first summarized to gene counts and then DESeq2 was used to convert count values to integer mode, correct for library size, and estimate dispersions and log2 fold changes between comparison groups. Genes with a Benjamini-Hochberg adjusted $p$-value $<0.05$ and log2 fold change $>+/-4$ between control and xenograft samples were considered significantly differentially expressed transcripts. Statistically differentially expressed canine genes were removed if they had a DESeq2 normalized value of greater than zero in the control (mouse sequences) as these would be genes that are highly homologous between the mouse and dog. Counts per million (CPM) values of genes were log2 transformed and mean centered prior to clustering. The ComplexHeatmap package was used for clustering and creating heatmap figures. Enriched pathway and functional classification analyses of differentially expressed transcripts were performed using QIAGEN's Ingenuity® Pathway Analysis (IPA®; QIAGEN, Redwood City, CA). The reference set for all IPA analyses was the Ingenuity Knowledge Base (genes only) and canine associated gene names were used as the output format for input datasets with canine genes and murine associated gene names were used as the output format for input datasets with murine genes.

qRT-PCR validation of sequencing data. Serum or plasma samples were obtained from client-owned dogs with naturally-occurring osteosarcoma before and after treatment as 
part of routine biobanking efforts. The samples included in the analysis were identified retrospectively. Serum samples were also obtained and biobanked from client-owned dogs that were hospitalized with various non-malignant conditions. Serum samples were obtained from staff- and student-owned dogs with no apparent disease. The samples were divided into a training set and a test set. The training set included dogs in one of four categories ("osteosarcoma”, "other neoplasia”, "non-neoplasia”, and "healthy" (dogs with no apparent disease)). Osteosarcoma dogs had a primary tumor of bone and were treatment naïve (Table S1). The test set included samples from dogs with osteosarcoma, after treatment ( $n=24$; Table S2). Blood was collected into vacutainer tubes that were centrifuged at 3,000 $\mathrm{xg}$ for 15 minutes. Aliquots of serum or plasma were transferred to $1.5 \mathrm{ml}$ microcentrifuge tubes and stored at $-80^{\circ} \mathrm{C}$ until analysis. All treatment decisions were at the discretion of the attending clinician. All procedures were approved by the Institutional Animal Care and Use Committees of The University of Minnesota under protocols 0802A27363, 1101A94713, 1312-31131A, 1504-32486A, 1702-34548A, 1803-35759A, and 2003-37952A and The Ohio State University 2010A0015-R2 and 2018A00000100. Exosomes were precipitated from canine serum or plasma samples using ExoQuick serum reagent (SBI, Palo Alto, CA, catalog \#EXOQ5A-1) according to the manufacturer's instructions. Additional steps were included for plasma samples: 10 $\mu$ l of thrombin (SBI, Palo Alto, CA, catalog \#TMEXO-1) was added for each $1 \mathrm{ml}$ of plasma. The sample was then mixed at room temperature for 5 minutes, followed by centrifugation at 10,000 rpm for 5 minutes. The supernatant was transferred to a new microcentrifuge tube, and the volume recovered was noted. Plasma and serum samples were subsequently treated the same. Briefly, the sample was mixed with ExoQuick reagent at a volume of $252 \mu$ ExoQuick per $1 \mathrm{ml}$ of serum. The mixture was incubated for 30 minutes at $4^{\circ} \mathrm{C}$, followed by centrifugation at $1,500 \times \mathrm{g}$ for 30 minutes to precipitate exosomes. The resulting supernatant was removed and discarded, and the tubes were centrifuged for an additional 5 minutes at $1,500 \times \mathrm{g}$ to remove any remaining supernatant. Exosomal RNA was extracted using the mirVana miRNA Isolation Kit (Ambion, Thermo Fisher Scientific, Waltham, MA), according to the manufacturer's instructions. Elimination of genomic DNA and reverse transcription were both carried out using QuantiTect Reverse Transcription Kit (Qiagen, 
Valencia, CA). Real-time quantitative reverse transcriptase PCR (qRT-PCR) was performed on a LIGHTCYCLER 96 (Roche, Indianapolis, IN) with FastStart SYBR Universal Green Master Mix (Roche, Indianapolis, IN) Protocol. GAPDH was used as the reference standard for normalization (14) and relative levels of steady state mRNA were established using the comparative [delta]Ct method. The relationship between RNA-sequencing data and qRT-PCR values for the transcripts of interest were analyzed using Pearson's correlation.

Machine learning._Gene expression data from samples of dogs with "no apparent disease" (Healthy, $n=13$ ), non-neoplastic/benign conditions treated with surgery (diseases other than cancer; $n=10)$, osteosarcoma $(n=27)$, and other neoplasia (nonosteosarcoma cancers; $n=2)$ ) pre-treatment samples (52 total) normalized to GAPDH (as internal control) were used to train and build different machine learning models. The normalized data were further transformed using three-component linear discriminant analysis (LDA). Different machine learning algorithms were then tested and compared to identify the top-performing predictive models that fit well with our data, including Logistic Regression (LR), Linear Discriminant Analysis (LDA), k-Nearest Neighbors (KNN), Decision Tree (CART), Gaussian Naïve Bayes (NB), Support Vector Machine (SVM), Bagging (BAG), Random Forest (RF), Extra Trees (EXT), Adaptive Boosting (ADA), Stochastic Gradient Boosting (SGB), Neural Network (NN), Ridge regression (RGD), and Stochastic Gradient Descent (SGD) classifiers Scikit-learn Python package (http://scikit-learn.sourceforge.net) (14). For training and optimization, the training dataset was randomly split into training and validation sets using k-fold cross-validations with sample stratification (when possible). k-fold cross-validation randomly splits data into k groups, where $\mathrm{k}$ - 1 groups were used for training and one remaining group was used for validation; repeated for $k$ times with each of $k$ validation sets being used only once. The k-fold cross-validation was then repeated and averaged across 100 iterations with random shuffling in between to ensure performance stability across multiple tests. For this study, a 10-fold cross-validation was used. Top models with the best averaged sensitivity and specificity were chosen for further optimization and testing. The sensitivity was calculated based on the ratio of True Positives / (True Positives + False 
Negatives) and the specificity was the ratio of True Negatives / (True Negatives + False Positives) (19). True positives were defined as the classification accuracy for osteosarcoma and true negatives were defined as the classified accuracy of nonosteosarcoma. Predictive power was also estimated for the final top-performing models based on their positive (PPV) and negative (NPV) predictive values. The PPV was the ratio of True Positives / (True Positives + False Positives) and NPV was the ratio of True Negatives / (True Negatives + False Negatives) (19). Data from the unknown samples (post-treatment osteosarcoma subjects) were transformed based on the fitted training set and classified using the top trained learning models. Results from the prediction calls were further tested against survival data of the post-treatment osteosarcoma subjects over time as a means for detecting residual disease.

Post-treatment osteosarcoma samples that were classified as "osteosarcoma" by all of the selected top machine learning models (either most accurate or most sensitive) were considered to be "osteosarcoma-detectable". Post-treatment osteosarcoma samples that received another classification by one or more of the top models were considered to be "osteosarcoma-NOT detectable". Kaplan-Meier survival analysis was performed using R packages survival (v3.27) and survminer (v0.48). A log-rank (Mantel-Cox) test was used to compare event-free survival times between dogs whose post-treatment samples were considered "osteosarcoma-detectable" and dogs whose post-treatment samples were considered "osteosarcoma-NOT detectable".

\section{Results}

\section{Exosome production by osteosarcoma cells is positively correlated with tumor}

aggressiveness. Previous studies have documented a quantitative relationship between tumor aggressiveness and the amount of exosomes produced (11). To investigate this in the context of osteosarcoma, the presence of tetraspanins CD9 and CD63, both of which are enriched in microvesicles, was quantified in 80 human 
osteosarcoma samples using immunohistochemistry (IHC) (Figs. S1A-B). The data show that stage-III tumors stained more robustly for both CD9 and CD63 than stage-I or stage-Il tumors (Figs. S1A-B), suggesting that a positive relationship between total detectable exosomes or exosomal protein and tumor stage also exists in osteosarcoma. To further delineate the functional involvement of exosomes in the progression of osteosarcoma, we sought to use a more tractable in vivo model system for exosome biomarker discovery. Given the molecular and biological similarities between human and canine osteosarcomas $(20,21)$, a spontaneous canine model was used for our studies. Our immediate next experiments, shown in the supplementary information, were thus devoted to characterizing canine osteosarcoma-derived exosomes and to confirm their conserved roles in the biology of the disease $(6,7)$. We first validated exosome production by canine osteosarcoma cell lines using immunofluorescence (IF). Previous experiments show that the biologic behavior of the primary tumors was conserved in the cell lines, with OSCA-40 being more aggressive $(12,17)$. Fig. S2 shows positive staining for CD81, CD63 and CD9 in secreted microvesicles from OSCA-40 and OSCA-32 canine osteosarcoma cell lines (Fig. S3B, and data not shown), indicating conservation of tetraspanins in extracellular vesicles, most likely representing exosomes, from humans and dogs.

To confirm the physical properties of canine osteosarcoma-derived extracellular vesicles as exosomes, and to validate their enrichment from cell lines and from serum, we utilized scanning electron microscopy (SEM), NanoSight particle tracking analysis, and immunoblotting (Figs. S3A-C and Fig. S4). SEM showed spherical microvesicles 
between 100 and $200 \mathrm{~nm}$ in diameter; this size and shape was consistent with that predicted for exosomes (Fig. S3A). Additionally, immunoblotting showed enrichment of CD63 and depletion of $\beta$-actin in the osteosarcoma cell line-derived exosomes relative to the whole cell lysates (Fig. S3B). Finally, nanoparticle tracking analysis showed that the mean vesicle size ranged from $149 \mathrm{~nm}-180 \mathrm{~nm}$ with a mode of $117 \mathrm{~nm}-132 \mathrm{~nm}$, (Fig. S3C). This range is similar to the microvesicle size determined by SEM and is also consistent with the expected size of exosomes (22-29). We also confirmed that exosomes enriched from serum samples of a dog with osteosarcoma and a dog with no evidence of disease were comparable in size to cell line-derived exosomes, as determined by SEM (Fig. S4). In all, these findings support the methodology used for exosome enrichment from cell lines and from serum samples.

\section{Osteosarcoma-derived exosomes can be internalized by stromal cells to} modulate gene expression and induce invasive cell behavior. The high propensity for distant metastatic growth in both human osteosarcoma patients and dogs with osteosarcoma has been well documented and is a key factor in survival rates $(1,2,30)$. The importance of exosomes in promoting a pre-metastatic niche has been characterized in pancreatic cancer $(31,32)$ as well as melanoma $(33)$. However, the ability of osteosarcoma exosomes to influence the complex cascade of events that occurs during metastasis, particularly their impact on stromal cells within the microenvironment, has not been fully addressed. The importance of cancer-associated fibroblasts and endothelial cells in tumor progression have been well described (34), making them a useful model system to investigate exosome internalization and 
influence on cell behavior. Ascertainment that secreted tumor-derived exosomes can be taken up by stromal cells in the organ that is the major target of metastasis requires a method to track tumor specific exosomes. This was accomplished by transfecting canine osteosarcoma cells with CD81 linked to a green fluorescent protein (GFP) tag.

Expression of the fusion protein in transfected OSCA-40 cells, and its incorporation into secreted microvesicles, were visualized using IF microscopy (Fig. S5A). Lung stromal fibroblasts and lung endothelial cells were selected as the most relevant target cells for analysis. Figs. S5B-E show that GFP-positive osteosarcoma-derived exosomes were internalized by human pulmonary fibroblasts or human pulmonary endothelial cells within 6 to $8 \mathrm{hr}$, with nearly $100 \%$ of the target cells showing GFP expression by $24 \mathrm{hr}$. The effect of internalized exosomes on the behavior of stromal fibroblasts and endothelial cells was addressed using cell migration and proliferation assays. A scratchfilling assay was performed to determine the migration activity of human pulmonary fibroblasts and endothelial cells following addition of osteosarcoma exosomes. An increase in cell number as well as target cell migration following addition of exosomes was apparent, with a more pronounced effect on proliferation (Figs. S6 A-B). To address the underlying mechanisms for these changes, we used a bead array to interrogate how osteosarcoma-derived exosomes altered gene expression in human pulmonary fibroblasts. The use of canine exosomes, human target cells, and a humanspecific bead array diminished the potential that we would detect canine mRNAs carried in the exosomes. The data show altered expression of genes mediating cell adhesion and motility, and inflammation (Figure S6C). 


\section{Serum-derived exosomal canine gene signatures identified in mouse xenografts}

are associated with osteosarcoma. The in vitro data suggested that exosomes influence the tumor microenvironment; however, it remained unclear if these studies are directly translatable to in vivo studies where tumor cells maintain a series of complex relationships within their local environment. To address the concerns of in vitro translatability, a previously described orthotopic xenograft mouse model was utilized $(12,17)$. Briefly, we established xenografts in nude mice using two canine osteosarcoma cell lines with different metastatic propensities, collected serum exosomes from these mice and from sham-treated controls (injected intratibially with PBS) and performed next-generation sequencing to characterize the full complement of exosomal mRNAs derived from the tumors, as well as from the host response (12). Predictably, no xenograft (canine) mRNAs were detectable in sera from the mice prior to tumor implantation, but canine mRNAs were readily apparent in sera from mice with established tumors (Fig. 1A). Interestingly, the exosomal transcripts identified in cultured canine osteosarcoma cells showed only minimal overlap (1.4\%) with the exosomal transcripts derived from the same canine cell lines when they formed tumors in vivo (data not shown), suggesting that the microenvironment is a major factor influencing exosome loading. Further analysis identified groups of canine exosomal mRNAs with correlation scores $>0.8$ that were part of canonical signaling pathways including cell death, cell signaling, metabolism, and immune response. Changes in the mRNA content of host exosomes were also detectable. Thirty-eight differentially expressed mouse mRNAs were identified in exosomes from animals bearing xenografts 
when compared to the sham-treated controls (Fig. 1B). These mRNAs were primarily associated with immune signaling and cellular metabolism (Fig. 1C), but a network of host mRNAs converging on TP53 was also enriched in exosomes from the tumorbearing mice (Fig. S7), suggesting that the implanted osteosarcomas created a strong stress response in the host.

\section{A five-gene "osteosarcoma-detectable" signature predicts the presence of} minimal residual disease in dogs with osteosarcoma after treatment. The absence of biomarkers to guide treatment is a major obstacle that has hindered progress in osteosarcoma therapy for dogs and humans alike. We believe that clusters of coexpressed exosomal mRNAs could provide such as biomarkers, independent of their biological function. Twenty-five canine mRNAs were reproducibly identifiable and highly expressed in tumor-derived serum exosomes (i.e., were always present in sera from mice with canine osteosarcoma tumors, but not in sera from sham-treated mice, Fig. 1A). To build a diagnostic biomarker set, we narrowed the list to the 10 mRNAs with the highest expression, with low inter-sample variation. Five of the 10 mRNAs, representing transcripts from the SKA2, NEU1, PAF1, PSMG2, and NOB1 genes were determined to be suitable candidates for the biomarker set by virtue of being detectable in serum exosomes derived from dogs with osteosarcoma.

"In-species validation" was done by evaluating abundance of these five exosomal mRNAs in 80 archival serum and/or plasma samples from 53 dogs divided into four groups. The clinical characteristics of enrolled dogs are shown in Supplemental Table 
S1. Serum or plasma samples were included as available in sample archives, and plasma samples were treated with the addition of thrombin to precipitate clotting factors; thereafter the samples were treated similarly, as detailed in the methods. Serum and plasma samples were available for simultaneous testing in a limited number of cases, and the results were concordant. The first group consisted of 28 samples from dogs with osteosarcoma. Of these, 26 included serum or plasma collected prior to and at various time points after treatment (amputation +/- chemotherapy) ranging from 2 to 984 days (median $=37$ ), one only included serum collected before treatment, and one only included serum collected after treatment. The second group consisted of 10 samples from dogs that had been diagnosed with non-neoplastic diseases. The third group consisted of two samples from dogs with intramedullary soft tissue sarcomas (metastatic carcinoma and hemangiosarcoma, the latter of which had pre- and posttreatment samples). And the fourth group consisted of 13 samples from dogs with no apparent disease, included as unaffected controls (henceforth referred to as "healthy") (Table S1).

Figs. S8A-E show the relative abundance of each of the five mRNAs in the biomarker set, measured by qRT-PCR, in serum exosomes from each subgroup of dogs; Fig. S8F shows the relative contribution of each of these mRNAs based on the F-values from the analysis of variance (ANOVA) as applied to the machine learning training algorithms described below. 
We used 53 samples that included those from "healthy" dogs, dogs with non-neoplastic diseases ("non-neoplasia”), dogs with intramedullary soft tissue sarcomas ("other neoplasia") and dogs with osteosarcoma prior to treatment ("pre-treatment osteosarcoma") as a training set to build machine learning models to identify dogs where osteosarcoma was present, and to distinguish osteosarcoma from the other conditions under test. Stratified 10 -fold cross-validation analysis of the training set was performed across 14 different machine learning algorithms based on the five-gene features combined with 3-component LDA-transformation, and repeated for 100 iterations with shuffling in between (Fig. 2). The top-performing models based on sensitivity and specificity were k-nearest neighbors (KNN), bagging (BAG), random forest (RF), and extra trees (EXT) classifiers (Fig. 2, red dashed boxes). The three most sensitive models were logistic regression (LR), linear discriminant analysis (LDA), and ridge (RDG) classifiers (Fig. 2, blue dashed boxes). The mean sensitivity (the prediction accuracy for "osteosarcoma") for the top-performing models ranged from $72-82 \%$, while the mean specificity ("non-osteosarcoma") was between $44 \%$ and $51 \%$. The lower specificity was largely due to poor classification of "non-neoplasia" and "other neoplasia" groups as compared to "healthy" and "osteosarcoma" (Fig. S9). When the prediction for "healthy" (i.e., dogs with no apparent disease) was analyzed independently, the mean specificity was indeed higher at around 60\% for the top four models (Fig. S9A), while the prediction accuracy for "non-neoplasia" and "other neoplasia" were only between 0 and $2 \%$ (Fig. S9B). While the mean specificities for the most sensitive models (LR, LDA, and RDG) were below $25 \%$, their mean sensitivities were $\geq 89 \%$. To show that the results were dependent on the relevant groups in the training set, group assignments 
were randomized. The machine learning performance was greatly affected following data randomization, showing a significant reduction in both sensitivity and specificity (Fig. S10).

The top-performing algorithms were chosen based on their sensitivity and specificity values for further validation (Fig. 3). The chosen top four algorithms (KNN, RF, BAG, and EXT) were then retested individually, by the Majority Rule voting approach, and by all-or-none calling method using 10 -fold cross-validation with 10 randomized iterations and summarized based on their predidictive power, shown was positive (PPV) and negative (NPV) predictive values for (Fig. 3A). LR, LDA, and RDG were chosen as the three most sentitive alogorithms and retested for their predictive power (Fig. 3B). We then utilized the post-treatment osteosarcoma samples $(n=24$; Table S2) to evaluate the ability of the "osteosarcoma-detectable" signature to predict the presence of minimal residual disease after treatment, and its relationship to event-free survival outcomes (i.e., duration of remission, Table S2). The data were analyzed to form Kaplan-Meier survival curves based on using the top-four performing algorithms and the three most sensitive algorithms (Figs. 4A-B). For this analysis, post-treatment osteosarcoma samples that were classified as "osteosarcoma" by all four models (either best performing based on sensitivity and specificity (Fig. 2, red dashed lines) or most sensitive (Fig. 2, blue dashed lines)) were considered to be "osteosarcoma detectable", and post-treatment osteosarcoma samples that received another classification by one or more of the four models were considered to be "osteosarcoma NOT detectable". Event-free survival (time to disease progression) was then compared 
between dogs whose post-treatment samples were considered "osteosarcoma detectable" and dogs whose post-treatment samples were considered "osteosarcoma NOT detectable". Fig. 4A shows that, using the top-four performing models, dogs with post-treatment samples predicted as "osteosarcoma - NOT detectable" had extended event-free survivals (median disease free interval of 371.1 days) compared to dogs with post-treatment samples predicted as "osteosarcoma-detectable" (median disease free interval of 149.0 days). The hazard ratio of "NOT detectable" versus "detectable" at 15 months was 2.252, $\mathrm{p}=0.1675(\mathrm{Cl}: 0.8557-5.927)$. Fig, 4B shows that, using the topthree most sensitive models, dogs with post-treatment samples predicted as "osteosarcoma - NOT detectable" had extended event-free survivals (median disease free interval of 722 days) compared to dogs with post-treatment samples predicted as "osteosarcoma-detectable" (median disease free interval of 215 days). The hazard ratio at 15 months of "NOT detectable" versus "detectable" was 3.066, $p=0.0398(\mathrm{Cl}: 1.054$ $-8.922)$

\section{Discussion}

Non-invasive tests that inform prognosis and longitudinal remission status remain a persistent unmet need for patients with osteosarcoma. In this study, we identified a gene signature associated with prognosis in canine osteosarcoma using a novel xenograft model and bioinformatics pipeline (12). We identified a 5-gene exosomal biomarker signature that was associated with prognosis following treatment in dogs with osteosarcoma, likely due to the presence of microscopic metastatic disease. The data 
demonstrate the robustness of our novel xenograft and bioinformatics platform to identify biomarkers for biologically or prognostically significant conditions.

Osteosarcoma, the most common primary tumor of bone, exhibits heterogeneous biological behavior (3-5); some tumors are extremely aggressive and unlikely to respond to conventional approaches, whereas others have more variable outcomes and may not require as aggressive treatment protocols. However, stratifying tumors based on aggressiveness is challenging in the clinical setting without suitable biomarkers. Previously identified transcriptional programs that predict tumor behavior and inform prognosis for osteosarcoma patients include the gene cluster expression summary score, or GCESS $(3,35)$. The GCESS methodology overcomes the challenge of tumor heterogeneity by identifying coordinately regulated transcripts that provide a cleaner signal, resulting in a biomarker set with greater sensitivity and specificity than that afforded by single biomarkers. However, the GCESS technique requires invasive tissue biopsies (17), so it has not been widely adopted in practice; additionally, its utility to monitor minimal residual disease is unknown. Therefore, non-invasive tests that inform prognosis and longitudinal remission status remain a persistent unmet need for patients with osteosarcoma. Development of a prognostic test could allow for patient stratification and development of improved personalized approaches in future clinical research, so that treatment plans minimize risk and maximize benefit, ultimately aiding in the development of new therapies optimized for relative risk. 
Extracellular vesicles, specifically exosomes, have great potential to be powerful diagnostic tools. In particular, they are stable in biological fluids, can be efficiently isolated, and contain cargo that is significantly associated with different disease states (9-11). The discovery of exosomes and their role in transferring genetic information between cells has sparked interest in utilizing these extracellular vesicles in the discovery of key genes promoting tumor progression $(32,33,36-38)$. However, the identification of cargo originating from diseased cells (the "signal") from the background of normal exosomes (the "noise") is still a major obstacle that precludes wide use of exosomes in clinical laboratory medicine. Even in the case of cancer where tumor cells release more exosomes than normal cells, the number of exosomes produced by the tumor is dwarfed by the exosomes produced by the patient's normal cells, masking all but the strongest tumor-derived exosome signals. Therefore, additional steps, such as sorting by flow cytometry or immunomagnetic enrichment with antibodies or tumor markers, are often undertaken to enrich specifically for cancer-associated exosomes $(39,40)$

The discovery platform described herein, utilizing canine osteosarcoma as an example, allows virtually complete separation of tumor-derived exosomal mRNA cargo and normal cell-derived exosomal mRNA cargo using osteosarcoma xenograft models and an innovative bioinformatics pipeline. Essentially, the mouse model filters the "noise" from the system and helps define tumor and host responses individually. The data from the xenograft models suggest that osteosarcoma-derived exosomes modify the metastatic niche, and host-derived exosomes create a window to understand the host 
response to the presence of tumors. Utilizing this platform, we developed a 5-gene signature associated with "presence of canine osteosarcoma," which was further validated in the relevant target species (dogs). The role that these mRNAs play in exosome biosynthesis or in intercellular communication is unclear. It is possible that when exosomes are taken up by cells at distant sites, these mRNAs could be translated in the target cells and contribute to molding the metastatic niche, potentially by immune modulation $(41,42)$. On the other hand, they might represent mRNAs that are eliminated from the tumor cells via exosomes because they are toxic when present in high abundance. Nevertheless, we determined that these five mRNAs would provide the foundation for an "osteosarcoma-detectable" signature that would be diagnostically useful. We decided to test the hypothesis that the "osteosarcoma-detectable" signature could be used in a machine learning environment to establish the presence of microscopic, minimal residual disease in dogs with osteosarcoma after surgery +/chemotherapy. Specifically, machine learning was applied to post-treatment serum samples obtained from dogs with osteosarcoma as a test-set for detection of minimal residual disease following treatment. The "osteosarcoma - not detectable" group had extended event-free survivals compared with the "osteosarcoma - detectable" group using either the top-performing or the most sensitive models, suggesting that the test was able to detect the presence of osteosarcoma cells (i.e., minimal residual disease) to prognosticate survival after initial treatment. Additional variables with the potential to introduce bias (including random assignment of samples to groups) were compared, and none generated a signature that resulted in robust cross validation after training. 
We hypothesize that dogs where the "osteosarcoma-detectable" signature was present after treatment had a shorter event-free survival due to the presence of minimal residual disease. Additional work is needed to validate the gene signature in an independent set of canine osteosarcoma serum samples. However, the ability to detect minimal residual disease using this signature suggests that it will have utility in the clinical setting for determining prognosis after treatment. This biomarker could be applied after surgery and/or the first round of conventional chemotherapy to guide the subsequent treatment of dogs with osteosarcoma and to alter the course of therapy as needed.

In conclusion, our data support the application of a novel platform consisting of an osteosarcoma xenograft model and bioinformatics pipeline, for discovery of prognostically significant, species-specific mRNAs. Moreover, our results document the utility of machine learning algorithms to enhance applicability of these mRNAs to address medical unmet needs, such as sensitive detection of minimal residual disease. Specifically, for this study we identified and validated a 5-gene signature associated with the presence of osteosarcoma in dogs. We further determined that this 5-gene signature obtained from serum exosomes, without the need for more invasive testing, was associated with prognosis, presumably due to the detection of minimal residual disease in dogs with osteosarcoma following treatment. This exosomal 5-gene signature could be applied to clinical veterinary practice and a comparable signature uncovered using our platform could be investigated in human osteosarcoma. Speciesappropriate signatures would allow for stratification of dogs and humans with 
osteosarcoma to minimize risk and maximize benefit of treatment, ultimately aiding in the development of novel therapies.

\section{Acknowledgments}

The authors would like to thank Mitzi Lewellen for assistance with the mouse work, Aaron Sarver and Don Bellgrau for manuscript review and discussion, Brenda Weigel, Mike Farrar, Daniel Vallera, Shruthi Naik, and Steven J. Russell for assistance with funding, and M. Gerard O'Sullivan and Ingrid Cornax for their assistance with mouse pathology. Additionally, we would like to thank Jong-Hyuk Kim, Ashley Schulte, Taylor DePauw, and Erin Dickerson for laboratory support and discussion, Jonah Cullen for assistance with figures, and Dayane Alcantara for technical assistance. Finally, we thank Dr. Holly Borghese of the OSU CVM Veterinary Biospecimen Repository and Blue Buffalo Veterinary Clinical Trials Office (BBVCTO) and Kathleen M. Stuebner, Sara Pracht, Kelly Bergsrud, Andrea Chehadeh, Amber Winter, and Donna Groschen of the Clinical Investigation Center $(\mathrm{CIC})$ of the University of Minnesota for their expertise in sample procurement.

\section{References}

1. Mirabello L, Troisi RJ, Savage SA. Osteosarcoma incidence and survival rates from 1973 to 2004: data from the Surveillance, Epidemiology, and End Results Program. Cancer. 2009;115(7):1531-43.

2. Allison DC, Carney SC, Ahlmann ER, Hendifar A, Chawla S, Fedenko A, et al. A meta-analysis of osteosarcoma outcomes in the modern medical era. Sarcoma. 2012;2012:704872. 
3. Scott MC, Temiz NA, Sarver AE, LaRue RS, Rathe SK, Varshney J, et al. Comparative Transcriptome Analysis Quantifies Immune Cell Transcript Levels, Metastatic Progression, and Survival in Osteosarcoma. Cancer Res. 2018;78(2):326-37. 4. Sarver AL, Thayanithy V, Scott MC, Cleton-Jansen AM, Hogendoorn PC, Modiano JF, et al. MicroRNAs at the human $14 q 32$ locus have prognostic significance in osteosarcoma. Orphanet J Rare Dis. 2013;8:7.

5. Scott MC, Sarver AL, Gavin KJ, Thayanithy V, Getzy DM, Newman RA, et al. Molecular subtypes of osteosarcoma identified by reducing tumor heterogeneity through an interspecies comparative approach. Bone. 2011;49(3):356-67.

6. Chicon-Bosch M, Tirado OM. Exosomes in Bone Sarcomas: Key Players in Metastasis. Cells. 2020;9(1).

7. Wolf-Dennen K, Kleinerman ES. Exosomes: Dynamic Mediators of Extracellular Communication in the Tumor Microenvironment. Adv Exp Med Biol. 2020;1258:189-97. 8. Ruivo CF, Adem B, Silva M, Melo SA. The Biology of Cancer Exosomes: Insights and New Perspectives. Cancer Res. 2017;77(23):6480-8.

9. Guo L, Guo N. Exosomes: Potent regulators of tumor malignancy and potential bio-tools in clinical application. Crit Rev Oncol Hematol. 2015;95(3):346-58.

10. Jia S, Zocco D, Samuels ML, Chou MF, Chammas R, Skog J, et al. Emerging technologies in extracellular vesicle-based molecular diagnostics. Expert Rev Mol Diagn. 2014;14(3):307-21.

11. Kalluri R. The biology and function of exosomes in cancer. J Clin Invest. 2016;126(4):1208-15.

12. Scott MC, Tomiyasu H, Garbe JR, Cornax I, Amaya C, O'Sullivan MG, et al. Heterotypic mouse models of canine osteosarcoma recapitulate tumor heterogeneity and biological behavior. Dis Model Mech. 2016;9(12):1435-44.

13. Scott M, Garbe, JR \& Modiano, JF. Identifying presence and composition of cellfree nucleic acids. . Patent application. 2016;(Regents of the University of Minnesota (Minneapolis, MN, US) USA

14. Yamini B, VanDenBrink PL, Refsal KR. Ovarian steroid cell tumor resembling luteoma associated with hyperadrenocorticism (Cushing's disease) in a dog. Vet Pathol. 1997;34(1):57-60.

15. Mather Q, Priego J, Ward K, Kundan V, Tran D, Dwivedi A, et al. A novel protein expression signature differentiates benign lipomas from well-differentiated liposarcomas. Mol Clin Oncol. 2017;7(3):315-21.

16. Modiano JF, Mayor J, Ball C, Fuentes MK, Linthicum DS. CDK4 expression and activity are required for cytokine responsiveness in T cells. $\mathrm{J}$ Immunol. 2000;165(12):6693-702.

17. Scott MC, Sarver AL, Tomiyasu H, Cornax I, Van Etten J, Varshney J, et al. Aberrant Retinoblastoma (RB)-E2F Transcriptional Regulation Defines Molecular Phenotypes of Osteosarcoma. J Biol Chem. 2015;290(47):28070-83.

18. GeneCopoeia Lentivirus Protocol 2018 [Available from: https://www.genecopoeia.com/wp-content/uploads/2018/03/Lentivirus-protocolGeneCopoeia.pdf.

19. Parikh R, Mathai A, Parikh S, Chandra Sekhar G, Thomas R. Understanding and using sensitivity, specificity and predictive values. Indian J Ophthalmol. 2008;56(1):4550 . 
20. Makielski KM, Mills LJ, Sarver AL, Henson MS, Spector LG, Naik S, et al. Risk Factors for Development of Canine and Human Osteosarcoma: A Comparative Review. Vet Sci. 2019;6(2).

21. Fenger JM, London CA, Kisseberth WC. Canine osteosarcoma: a naturally occurring disease to inform pediatric oncology. ILAR J. 2014;55(1):69-85.

22. Ludwig N, Whiteside TL, Reichert TE. Challenges in Exosome Isolation and Analysis in Health and Disease. Int J Mol Sci. 2019;20(19).

23. Sokolova V, Ludwig AK, Hornung S, Rotan O, Horn PA, Epple M, et al. Characterisation of exosomes derived from human cells by nanoparticle tracking analysis and scanning electron microscopy. Colloids Surf B Biointerfaces.

$2011 ; 87(1): 146-50$.

24. Slomka A, Urban SK, Lukacs-Kornek V, Zekanowska E, Kornek M. Large Extracellular Vesicles: Have We Found the Holy Grail of Inflammation? Front Immunol. 2018;9:2723.

25. Ricklefs FL, Maire CL, Reimer R, Duhrsen L, Kolbe K, Holz M, et al. Imaging flow cytometry facilitates multiparametric characterization of extracellular vesicles in malignant brain tumours. J Extracell Vesicles. 2019;8(1):1588555.

26. Banfai K, Garai K, Ernszt D, Pongracz JE, Kvell K. Transgenic Exosomes for Thymus Regeneration. Front Immunol. 2019;10:862.

27. Kowal J, Arras G, Colombo M, Jouve M, Morath JP, Primdal-Bengtson B, et al. Proteomic comparison defines novel markers to characterize heterogeneous populations of extracellular vesicle subtypes. Proc Natl Acad Sci U S A. 2016;113(8):E968-77.

28. Li N, Huang Z, Zhang X, Song X, Xiao Y. Reflecting Size Differences of Exosomes by Using the Combination of Membrane-Targeting Viscosity Probe and Fluorescence Lifetime Imaging Microscopy. Anal Chem. 2019;91(23):15308-16. 29. Liu F, Vermesh O, Mani V, Ge TJ, Madsen SJ, Sabour A, et al. The Exosome Total Isolation Chip. ACS Nano. 2017;11(11):10712-23.

30. Jeffree $\mathrm{GM}$, Price $\mathrm{CH}$, Sissons HA. The metastatic patterns of osteosarcoma. $\mathrm{Br}$ J Cancer. 1975;32(1):87-107.

31. Jung T, Castellana D, Klingbeil P, Cuesta Hernandez I, Vitacolonna M, Orlicky DJ, et al. CD44v6 dependence of premetastatic niche preparation by exosomes. Neoplasia. 2009;11(10):1093-105.

32. Costa-Silva B, Aiello NM, Ocean AJ, Singh S, Zhang H, Thakur BK, et al. Pancreatic cancer exosomes initiate pre-metastatic niche formation in the liver. Nat Cell Biol. 2015;17(6):816-26.

33. Peinado H, Aleckovic M, Lavotshkin S, Matei I, Costa-Silva B, Moreno-Bueno G, et al. Melanoma exosomes educate bone marrow progenitor cells toward a prometastatic phenotype through MET. Nat Med. 2012;18(6):883-91.

34. Langsten KL, Kim JH, Sarver AL, Dewhirst M, Modiano JF. Comparative Approach to the Temporo-Spatial Organization of the Tumor Microenvironment. Front Oncol. 2019;9:1185.

35. Lesluyes T, Delespaul L, Coindre JM, Chibon F. The CINSARC signature as a prognostic marker for clinical outcome in multiple neoplasms. Sci Rep. 2017;7(1):5480. 
36. Melo SA, Sugimoto H, O'Connell JT, Kato N, Villanueva A, Vidal A, et al. Cancer exosomes perform cell-independent microRNA biogenesis and promote tumorigenesis. Cancer Cell. 2014;26(5):707-21.

37. Pan BT, Teng K, Wu C, Adam M, Johnstone RM. Electron microscopic evidence for externalization of the transferrin receptor in vesicular form in sheep reticulocytes. J Cell Biol. 1985;101(3):942-8.

38. Garnica TK, Lesbon JCC, Avila A, Rochetti AL, Matiz ORS, Ribeiro RCS, et al. Liquid biopsy based on small extracellular vesicles predicts chemotherapy response of canine multicentric lymphomas. Sci Rep. 2020;10(1):20371.

39. Theodoraki MN, Hoffmann TK, Whiteside TL. Separation of plasma-derived exosomes into CD3((+)) and CD3((-)) fractions allows for association of immune cell and tumour cell markers with disease activity in HNSCC patients. Clin Exp Immunol. 2018;192(3):271-83.

40. Melo SA, Luecke LB, Kahlert C, Fernandez AF, Gammon ST, Kaye J, et al. Glypican-1 identifies cancer exosomes and detects early pancreatic cancer. Nature. 2015;523(7559):177-82.

41. Troyer RM, Ruby CE, Goodall CP, Yang L, Maier CS, Albarqi HA, et al. Exosomes from Osteosarcoma and normal osteoblast differ in proteomic cargo and immunomodulatory effects on T cells. Exp Cell Res. 2017;358(2):369-76.

42. Pu F, Chen F, Zhang Z, Liu J, Shao Z. Information Transfer and Biological Significance of Neoplastic Exosomes in the Tumor Microenvironment of Osteosarcoma. Onco Targets Ther. 2020;13:8931-40. 


\section{Figure legends}

Figure 1. Detecting biomarkers of disease and host response. Heatmap of 25 differentially expressed dog transcripts $(A)$ and 38 differentially expressed mouse transcripts (B) identified by statistical testing with 'DESeq2'. Colored toe bars represent the different experimental samples. Asterisks in (A) indicate the genes incorporated into the osteosarcoma gene signature. The color coded (red to blue) scale represents +/change gene expression. (C) Pathways identified by Ingenuity ${ }^{\circledR}$ Pathway Analysis as being associated with differentially expressed host (mouse) genes.

Figure 2. Machine learning model comparison based on repeated stratified 10-fold cross-validation analysis of the LDA-transformed training set. Boxplot of machine learning performance across different models tested shown as sensitivity and specificity based on 100 repeated 10 -fold cross-validation. Red dashed boxes indicate the top four models with the highest performance, and blue dashed boxes indicate the top three most sensitive models. Sensitivity: proportion of selecting true osteosarcoma; specificity: proportion of selecting non-osteosarcoma (Healthy, Other neoplasia, and Non-neoplasia).

Figure 3. Positive predictive value and negative predictive values. Positive predictive (PPV) and negative predictive (NPV) values (shown as \%) based on 10 iterations of shuffled 10 -fold cross-validation for (A) the top four learning models (KNN, BAG, RF, and EXT) and (B) the three most sensitive models (LR, LDA, and RDG). PPV and NPV are also shown for a combined prediction of the top four models shown in $A$ based on the Majority Rule voting (VOTE) and by all-or-none prediction, where Osteosarcoma is called only if agreed by all models (ALL), otherwise the prediction is called as nonOsteosarcoma.

Figure 4. Machine learning models predict presence minimal residual disease in canine osteosarcoma. Post-treatment samples (test set) from dogs with osteosarcoma $(n=24)$ were classified as "osteosarcoma - detectable" or "osteosarcoma - not detectable" based on predictions from $(A)$ the four best performing machine learning models $(K N N$, $B A G, R F$, and EXT), or (B) the three most sensitive machine learning models (LR, LDA, RDG). Kaplan-Meier survival curves demonstrating time to relapse for subset of dogs with osteosarcoma with available survival data, comparing those whose post-treatment samples were classified as "osteosarcoma - detectable" with those whose posttreatment samples were classified as "osteosarcoma - not detectable", (A) $p=0.1001$; (B) $p=0.0398$. 
bioRxiv preprint doi: https://doi.org/10.1101/2021.02.11.429432; this version posted February 12. 2021. The copyright holder for this preprint (which was not certified by peer review) is the author/funder, who has granted bioRxiv a license to display the preprint in perpetuity. It is made available under aCC-BY-NC-ND 4.0 International license.

\section{Abstract}

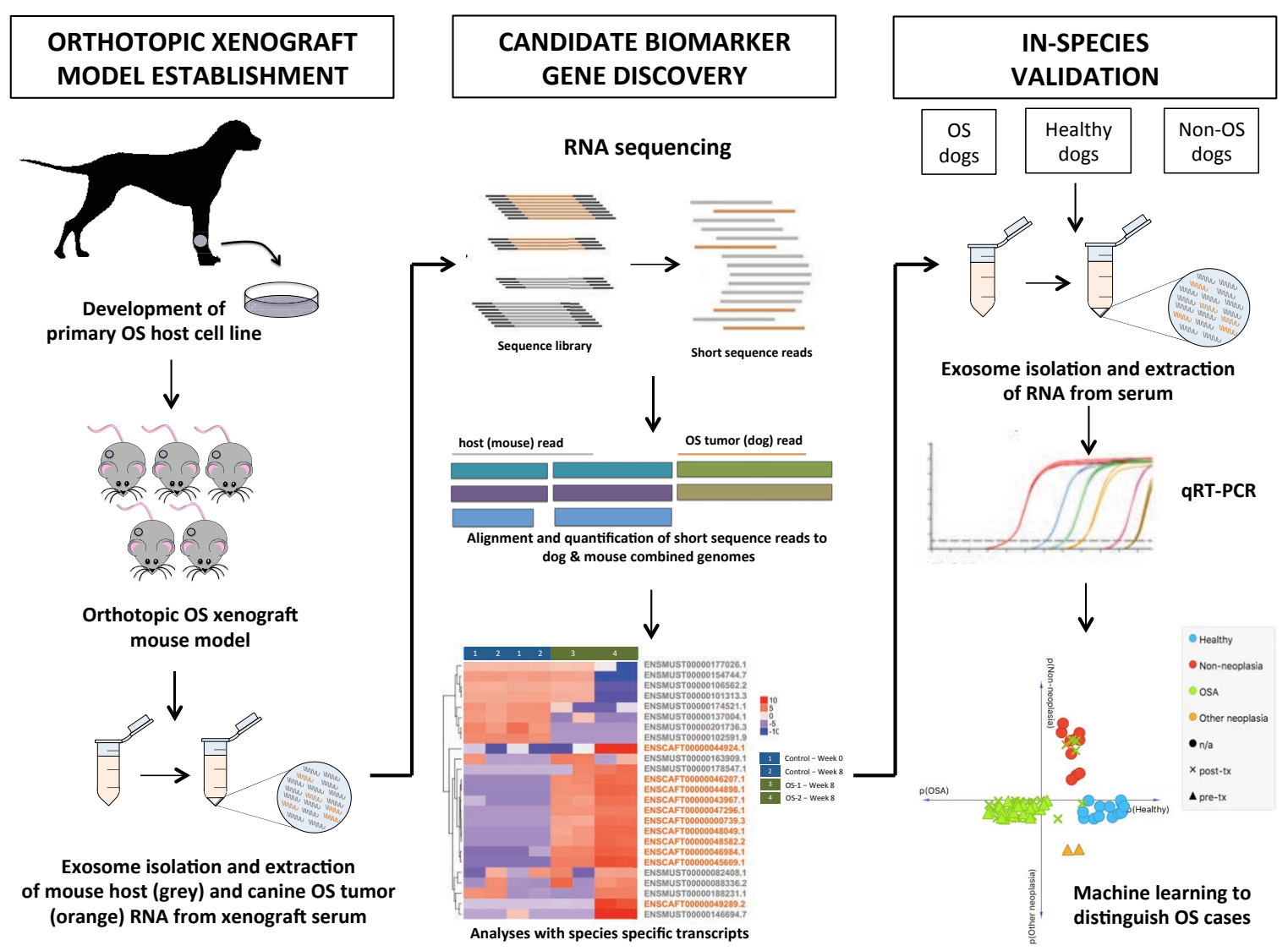


bioRxiv preprint doi: https://doi.org/10.1101/2021.02.11.429432; this version posted February 12, 2021. The copyright holder for this preprint (which was not certified by peer review) is the author/funder, who has granted bioRxiv a license to display the preprint in perpetuity. It is made available under aCC-BY-NC-ND 4.0 International license.

\section{Figure 1}
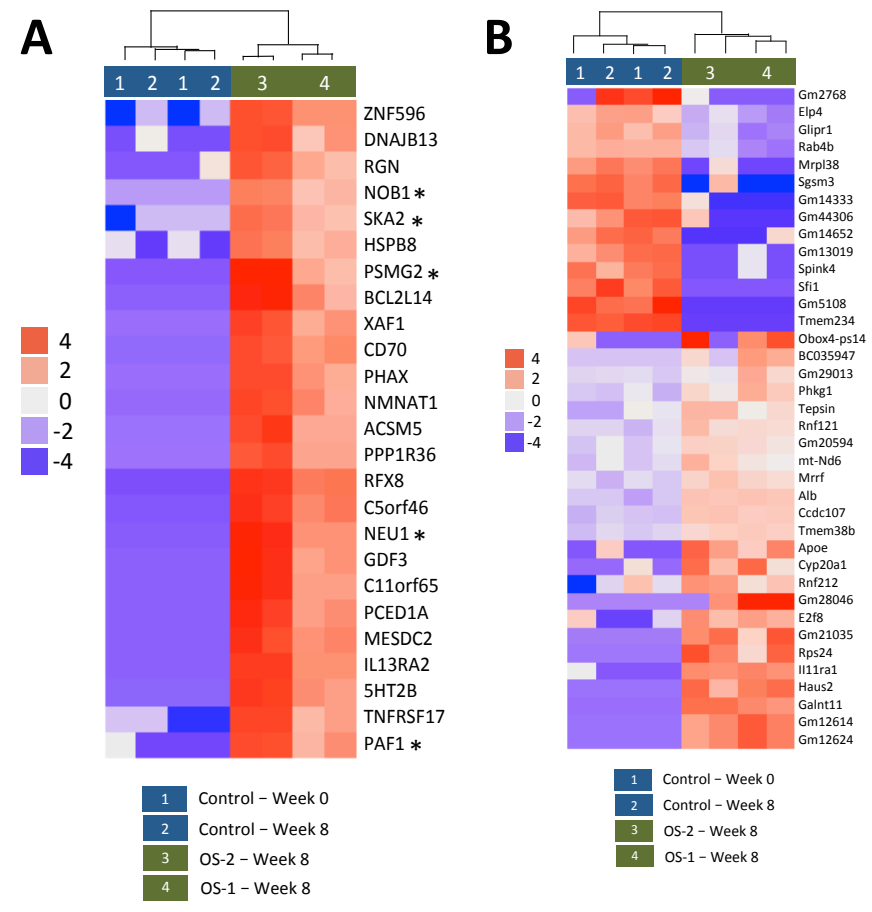

C

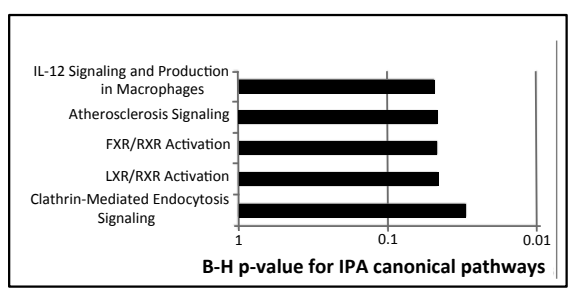

Figure 1. Detecting biomarkers of disease and host response. Heatmap of 25 differentially expressed dog transcripts (A) and 38 differentially expressed mouse transcripts (B) identified by statistical testing with 'DESeq2'. Colored toe bars represent the different experimental samples. Asterisks indicate the genes incorporated into the osteosarcoma gene signature. The color coded (red to blue) scale represents + - change gene expression. (C) Pathways identified by Ingenuity ${ }^{\circledR}$ Pathway Analysis as being associated with differentially expressed host (mouse) genes. 


\section{Figure 2}

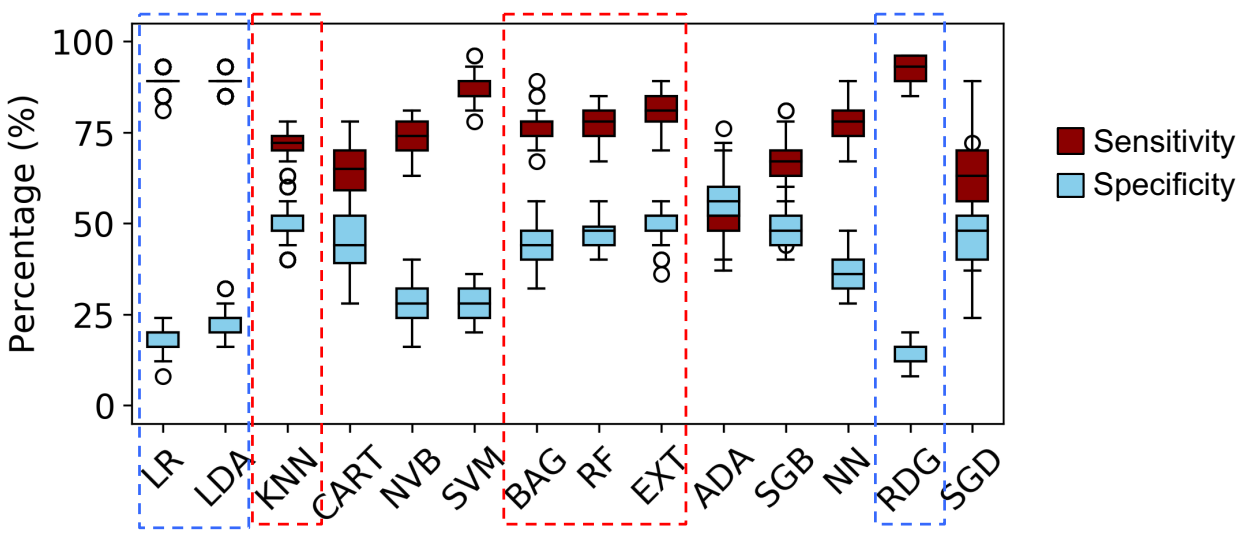

Figure 2. Machine learning models comparison based on repeated stratified 10-fold cross-validation analysis of the LDA-transformed training set. Boxplot of machine learning performance across different models tested shown as sensitivity and specificity based on 100 repeated 10 -fold cross-validation. Red dashed boxes indicate the top four models with the highest performance, and blue dashed boxes indicate the top three most sensitive models. Sensitivity: proportion of selecting true osteosarcoma; specificity: proportion of selecting non-osteosarcoma (Healthy, Other neoplasia, and Non-neoplasia). 


\section{Figure 3}

A

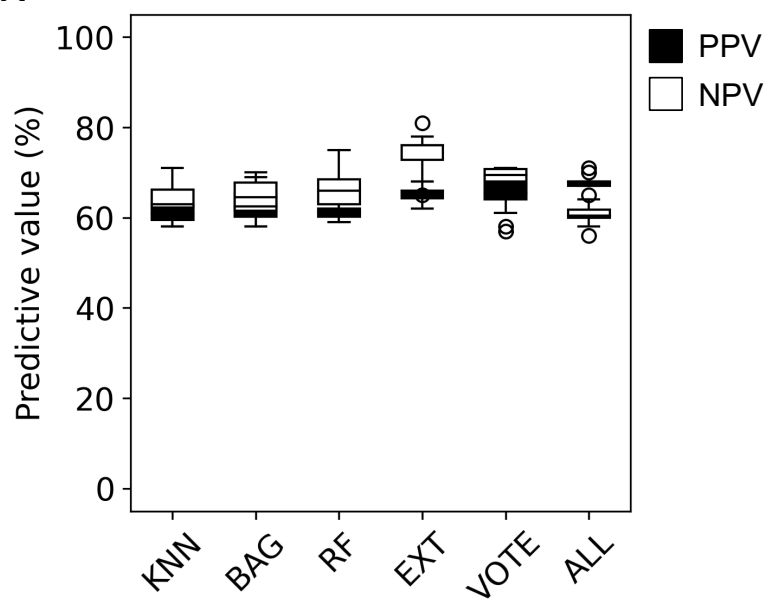

B

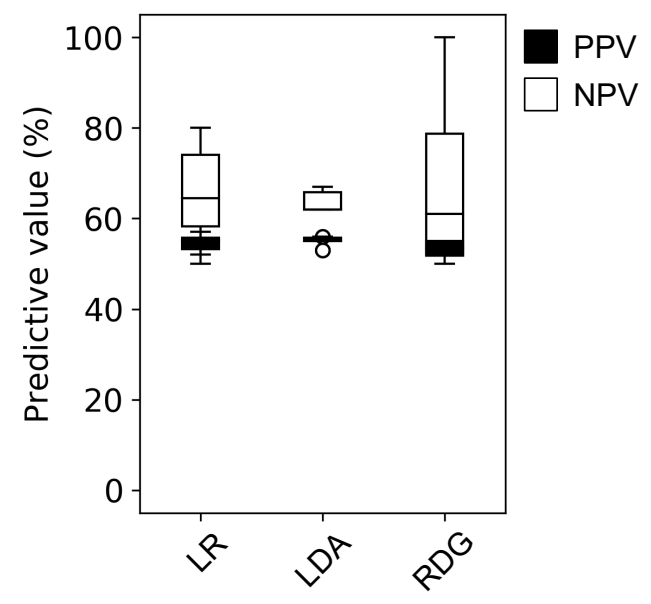

Figure 3. Positive predictive value and negative predictive values. Positive predictive (PPV) and negative predictive (NPV) values (shown as \%) based on 10 iterations of shuffled 10-fold cross-validation for (A) the top four learning models (KNN, BAG, RF, and EXT) and (B) the three most sensitive models (LR, LDA, and RDG). PPV and NPV are also shown for a combined prediction of the top four models shown in A based on the Majority Rule voting (VOTE) and by all-or-none prediction, where Osteosarcoma is called only if agreed by all models $(A L L)$, otherwise the prediction is called as non-Osteosarcoma. 


\section{Figure 4}

Figure 4. Machine learning models predict presence minimal residual disease in canine osteosarcoma. Posttreatment samples (test set) from dogs with osteosarcoma $(n=24)$ were classified as "osteosarcoma - detectable" or "osteosarcoma - not detectable" based on predictions from (A) the four best performing machine learning models (KNN, BAG, RF, and EXT), or (B) the three most sensitive machine learning models (LR, LDA, RDG). Kaplan-Meier survival curves demonstrating time to relapse for subset of dogs with osteosarcoma with available survival data, comparing those whose post-treatment samples were classified as "osteosarcoma - detectable" with those whose post-treatment samples were classified as "osteosarcoma - not detectable", (A) $p=0.1001$; (B) $p=0.0398$.

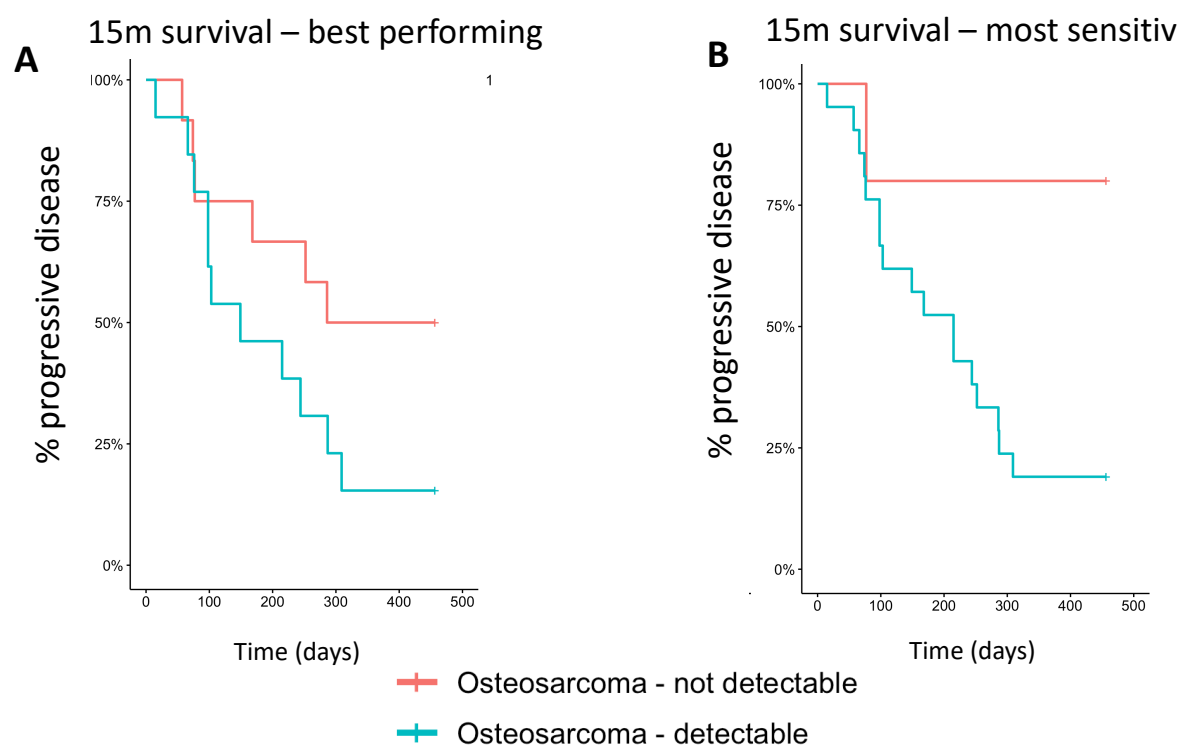

